\section{Long-term neuropsychological sequelae, emotional wellbeing and quality of life in patients with acquired thrombotic thrombocytopenic purpura}

\author{
Silvia Riva, ${ }^{1,2^{*}}$ Ilaria Mancini, ${ }^{3 *}$ Alberto Maino, ${ }^{1,4}$ Barbara Ferrari, ${ }^{1}$ \\ Andrea Artoni, ${ }^{1}$ Pasquale Agosti ${ }^{3}$ and Flora Peyvandi ${ }^{1,3}$
}

${ }^{1}$ Fondazione IRCCS Ca' Granda Ospedale Maggiore Policlinico, Angelo Bianchi Bonomi Hemophilia and Thrombosis Center, Milan, Italy; ${ }^{2}$ St Mary's University, Twickenham, London, UK; '3niversità degli Studi di Milano, Department of Pathophysiology and Transplantation, and Fondazione Luigi Villa, Milan, Italy and ${ }^{4}$ Azienda Provinciale per $\mathrm{i}$ Servizi Sanitari, Internal Medicine Unit, Trento, Italy

*SR and IM contributed equally as co-first authors.

\section{ABSTRACT}

$\mathrm{N}$ eurological symptoms related to microthrombosis are the hallmark of acute manifestations of acquired thrombotic thrombocytopenic purpura (TTP). Despite the achievement of hematological remission, patients may report persisting neurological impairment that affects their quality of life. To assess the long-term neuropsychological consequences of acute TTP, we recruited 35 acquired TTP patients (77\% females, median age at onset 41 years, interquartile range: $35-48$ ) regularly followed at our outpatient clinic of thrombotic microangiopathies in Milan (Italy) from December 2015 to October 2016. Patients underwent a psychological evaluation of memory and attentional functions, emotional wellbeing and health-related quality of life at least three months after their last acute TTP event (median 36 months, interquartile range: 17-54). During the psychological consultation, 17 patients (49\%) referred persisting subjective neurological impairment in the frame of a remission phase, with at least one symptom as disorientation, loss of concentration, dizziness, lack of balance, headache and diplopia. Neuropsychological assessment revealed lower scores than the Italian general population pertaining to direct, indirect and deferred memory. A higher degree of impairment of memory domains was found in patients with neurological involvement at the time of presentation of the first acute TTP episode. Anxiety and depression were detected in seven (20\%) and 15 $(43 \%)$ patients, respectively. Health-related quality of life was lower than the Italian general population, with mental domains more impacted than physical domains (mean difference 58.43, 95\% confidence interval: 71.4945.37). Our study demonstrates compromised memory and attention functions, persisting anxiety/depression symptoms and a generally reduced quality of life in patients recovering from acute acquired TTP. New clinical strategies should be considered to improve these symptoms.

\section{Introduction}

Thrombotic thrombocytopenic purpura (TTP) is a rare multisystem microangiopathy with fluctuating signs and symptoms. Its mortality rate can reach $90 \%$ when patients are left untreated, but it is reduced to $10 \%$ if patients are properly treated in the first 24 hours after diagnosis. Recovery is usually complete, but with a risk of relapse and the uncommon occurrence of persistent neurologic, cardiac and renal abnormalities. ${ }^{1}$ After recovery, despite normal physical examination and laboratory data, many patients complain of difficulties with memory, headache, loss of concentration and endurance, as expressed at patient support group meetings. Some attempts to quantify those neurological deficits in acquired TTP patients have been made in the context of small observational studies, but without

Ferrata Storti Foundation

Haematologica 2020
Volume 105(7):1957-1962

\section{Correspondence:}

FLORA PEYVANDI

flora.peyvandi@unimi.it

Received: May 17, 2019.

Accepted: September 19, 2019.

Pre-published: September 26, 2019.

doi:10.3324/haematol.2019.226423

Check the online version for the most updated information on this article, online supplements, and information on authorship \& disclosures: www.haematologica.org/content/105/7/1957

\section{(C)2020 Ferrata Storti Foundation}

Material published in Haematologica is covered by copyright. All rights are reserved to the Ferrata Storti Foundation. Use of published material is allowed under the following terms and conditions:

https://creativecommons.org/licenses/by-nc/4.0/legalcode. Copies of published material are allowed for personal or internal use. Sharing published material for non-commercial purposes is subject to the following conditions: https://creativecommons.org/licenses/by-nc/4.0/legalcode, sect. 3. Reproducing and sharing published material for commercial purposes is not allowed without permission in writing from the publisher. 
taking into account a whole assessment of cognitive, emotional and health-related quality of life (HrQoL) dimensions. ${ }^{2-3}$ Cognitive domains required for complex attention, concentration skills and high level memory functions may be involved in patients with TTP due to diffuse microvascular subcortical lesions, similarly to neurologically normal individuals with untreated hypertension, sickle cell disease and multi-infarct dementia. Two widely accepted measures to evaluate $\mathrm{HrQoL}$ are the Short-Form $36(\mathrm{SF}-36)^{4}$ and the EuroOol 5D (EQ-5D). ${ }^{5}$ They are selfreported scales providing a numerical score to identify the level of perceived health status. For their generic nature, the SF-36 and the EQ-5D are frequently used in chronic conditions (e.g. hemophilia) and are applicable to many diseases.

With this background and gaps of knowledge, we set up a study in order to investigate persistent cognitive abnormalities, emotional wellbeing and quality of life in patients who had recovered from an acute episode of acquired TTP. We also analyzed whether or not the presence of neurological involvement during the acute phase of TTP or severe ADAMTS13 deficiency during disease remission were related to persistent neurocognitive defects. Finally, we investigated whether there was an association between the emotional status of the patients and their quality of life.

\section{Methods}

\section{Patients}

We performed a cross-sectional study of 35 patients with acquired TTP regularly followed at our out-patient clinic of thrombotic microangiopathies in Milan (Italy). Patients were enrolled at least three months after their last acute TTP event (median time of 36 months, interquartile range [IOR]: 17-54) from December 2015 to October 2016, when they underwent a comprehensive neuropsychological evaluation including memory and attentional functions, emotional wellbeing and HrQoL. Demographic and clinical variables were recorded, including age, sex, ethnicity, job status, level of education, clinical and biochemical data at the time of acute TTP (neurological involvement, platelet count and haemoglobin level at presentation, number of plasma exchange procedures required to attain remission), and plasma ADAMTS13 activity levels at the time of the neuropsychological assessment $( \pm$ three months). Enrolment criteria are described in the Online Supplementary Table S1.

Written informed consent was obtained from all subjects with approval of the Ethics Committee of Fondazione IRCCS Ca' Granda Ospedale Maggiore Policlinico, in accordance with the Declaration of Helsinki.

\section{Neurocognitive, emotional and HrQoL assessments}

Neurocognitive and psychological assessments were administered by a board-certified psychologist in the standardized fashion in the frame of a single assessment session, which required approximately 1 hour to be completed, and included a test battery measuring two major cognitive domains, memory and attention, ${ }^{6-}$ ${ }^{8}$ the Hamilton Depression (HAM-D) ${ }^{9}$ and Anxiety (HAM-A $)^{10}$ rating scales for emotional wellbeing, and the Short-Form (SF) 364 form for HrQoL (Online Supplementary Material and Methods and Online Supplementary Table S2).

\section{Statistical analysis}

Descriptive statistics were used for demographic, clinical and laboratory characteristics. Categorical variables were expressed as counts and percentages, continuous variables as means or medians with standard deviation (SD) or IOR. With regards to neuropsychological and HrQoL analyses, each subject's raw score on each test was converted to a standardized score based on normative data generated from the value of the normal population according to the subject's age and education level, as appropriate. ${ }^{11-15}$ Standardized scores of TTP patients were then compared with norm-referenced data from the Italian population $^{11-15}$ by calculating the difference of means with $95 \%$ confidence intervals (CI) using unpaired and paired t-tests. Similarly, difference of means with $95 \%$ CI from unpaired and paired $t$ tests were used to compare neurocognitive assessment results in acquired TTP patients with and without neurological manifestations during the first acute episode of TTP, and with and without reduced ADAMTS13 activity during disease remission, close to the neuropsychological evaluation. For this analysis, an ADAMTS13 activity cut-off of $45 \%$ was used (i.e. the lower limit of the normality range in our ADAMTS13 activity assays). With regards to HrQoL, a standardized score of 50 was considered the cut-off for an acceptable quality of life. ${ }^{15-16}$ Finally, nonparametric correlation analyses were performed to evaluate the relationship between the results of emotional wellbeing tests and those of neurocognitive assessments or aggregated HrQoL scales.

Statistical analyses were performed by SPSS, release 25.0 (IBM Corp., Armonk, NY, USA), and GraphPad Prism, version 7.03 (GraphPad Software, La Jolla, CA, USA).

\section{Results}

Between December 2015 and October 2016, 41 acquired TTP patients were approached for participating in the study during a follow-up visit at our out-patient clinic of thrombotic microangiopathy. Of them, one patient refused to participate and one patient was excluded owing to a pre-existing psychiatric disease. Four were not constantly attended at our center, and therefore they were excluded from the study. Thus, 35 patients were included in the study and underwent psychological tests and neurocognitive examinations (see the Online Supplementary Material and Methods). Patient characteristics are reported in Table 1. All but one patient were Caucasian, with a female to male ratio of about $3: 1$ and a median age at TTP onset of 41 years (IOR: $35-48)$. At the time of neuropsychological evaluation, $10(29 \%)$ of 35 patients had suffered from recurrent TTP bouts. 22 patients $(63 \%)$ presented with neurological signs and symptoms at presentation of the first acute TTP episode (including coma [ $\mathrm{n}=2]$, focal neurological signs [ $\mathrm{n}=12]$, personality changes $[\mathrm{n}=2]$, transient ischemic attack $[n=4]$, seizures $[n=1]$, stroke $[n=3])$. During the psychological consultation, 17 (49\%) patients reported persisting subjective neurological impairment in the remission phase, with at least one symptom as disorientation, loss of concentration, dizziness, lack of balance (unable to control and maintain the body position all the time) headache, and diplopia.

\section{Results of neurocognitive assessment}

At the digit span test, $25(71 \%)$ and $23(66 \%)$ patients had a scoring lower than the mean of the general population in direct (mean difference -1.26 ; 95\% CI: -1.64$0.87]$ ) and backward (mean difference -1.49 ; 95\% CI: - 
2.02--0.96) memory, respectively (Table 2). Similarly, lower scores in TTP patients were observed in the Rey List tests for both direct (mean difference -5.87 ; 95\% CI: 8.57- -3.17) and deferred memory (mean difference -1.67; 95\% CI: -2.32--1.02).

With regards to the attention domain, TTP patients were slower in performing the trail making B test (sustained and divided attention) in comparison with the general population (mean difference 65.09 seconds; 95\% CI: 47.23-82.94). Conversely, patients were slightly faster in performing the trail making A test, which measures focused attention (mean difference -10.63 seconds; 95\% CI: 15.81--5.44).

When we analyzed scores of neurocognitive assessments in patients with and without neurological signs and symptoms at presentation of the first acute TTP episode, we observed a higher degree of impairment in the memory domains of the first group of patients in 3 of 4 memory tests (digit span [direct]: mean difference -0.78 ; 95\% CI: -1.54--0.02; digit span [backward]: mean difference 0.90 ; 95\% CI: -1.96--0.17]; Rey word list [deferred]: mean difference -1.39; 95\% CI: -2.65--0.13) (Table 3).

No differences in neuropsychological assessments were found between patients with ADAMTS13 activity levels during remission below and above $45 \%$ (Table 4).

\section{Results of emotional assessment}

TTP patients presented a mean level of anxiety with the HAM-A of $9.6(\mathrm{SD}=8.1)$ and a mean level of depression with the HAM-D of $7.4(\mathrm{SD}=5.7)$. The presence of clinical anxiety (HAM-A score $>13$ ) was detected in seven $(20 \%)$ of interviewed patients, while the presence of clinical depression (HAM-D score >7) was present in 15 $(43 \%)$ of them. All seven patients with clinical anxiety presented concomitant clinical depression. Five (14\%) patients showed a severe anxiety (HAM-A score $>24$ ) and five $(14 \%)$ a medium level of depression (HAM-D score $>18$ ). No patients presented severe levels of depression (HAM-D score $>24$ ).

Among the type of disturbances, we found that the most impaired domains in HAM-D were "work activities" ( $n=10,77 \%)$, "depressed mood" ( $n=8,60 \%)$ and "early insomnia" ( $n=4,27 \%$ ) while in HAM-A there were "intellectual symptoms" (i.e. difficulty in concentration and poor memory) ( $n=682 \%)$ and "tension" ( $n=4,54 \%)$.

At correlation analysis, better wellbeing was associated with better memory function (the sign of the correlation coefficient is negative because of the opposite interpretation scale of the two measurements): HAM-A test versus: digit span direct Spearman rho -0.472, $P=0.004$; digit span backward Spearman rho -0.597, $P<0.001$; Rey list direct Spearman rho 0.310, $P=0.075$; Rey list recall: Spearman rho $-0.432, P=0.011$; HAM-D test versus: digit span direct Spearman rho $-0.474, P=0.004$; digit span backward Spearman rho -0.594, $P<0.001$; Rey list direct Spearman rho 0.357, $P=0.038$; Rey list recall: Spearman rho -0.499 , $P=0.003$.

\section{Results of HrQoL assessment}

Table 5 displays the mean scores of the SF-36 assessments for each of the eight domains by physical and mental components: physical activity (PA), role physically $(\mathrm{RP})$, bodily pain $(\mathrm{BP})$, general health $(\mathrm{GH})$, vitality $(\mathrm{VI})$, social functioning (SF), role emotional (ER) and mental health $(\mathrm{MH})$. Acquired TTP patients showed lower nor-
Table 1. Demographic and clinical characteristics of 35 thrombotic thrombocytopenic purpura patients included in the study. Clinical and laboratory data pertain the first acute thrombotic thrombocytopenic purpura episode.

\begin{tabular}{|c|c|}
\hline Characteristics & $\begin{array}{l}\text { TIP patients } \\
(n=35)\end{array}$ \\
\hline \multicolumn{2}{|l|}{ Demographic data } \\
\hline Male, n (\%) & $8(23)$ \\
\hline Caucasian, n (\%) & $34(97)$ \\
\hline Age at TTP onset, years, median (IQR) & $41(35-48)$ \\
\hline $\begin{array}{l}\text { Age at neuropsychological evaluation, } \\
\text { years, median (IQR)* }\end{array}$ & $45(39-55)$ \\
\hline Mean school level, years & 13 \\
\hline Job status - workers, n (\%) & $30(77)$ \\
\hline \multicolumn{2}{|l|}{ Clinical characteristics at the first acute TTP episode } \\
\hline Neurological involvement, n (\%) & $22(63)$ \\
\hline Platelet count, x $10^{9} / \mathrm{L}$, median $(\mathrm{IQR})^{\dagger}$ & $13(8-27)$ \\
\hline Hemoglobin, $\mathrm{g} / \mathrm{dL}$, median $(\mathrm{IQR})^{\dagger}$ & $7.8(6.8-10.0)$ \\
\hline Number of PEX to attain remission, median $(\mathrm{IQR})^{\dagger}$ & $11(6-20)$ \\
\hline \multicolumn{2}{|l|}{$\begin{array}{l}\text { Laboratory parameters close to the } \\
\text { neuropsychological evaluation }\end{array}$} \\
\hline Platelet count, x 109/L, median (IQR) & $251(212-297)$ \\
\hline Hemoglobin, g/dL, median (IQR) & $13.4(12.8-14.3)$ \\
\hline \multicolumn{2}{|l|}{ ADAMTS13 activity close to neuropsychological evaluation ${ }^{\dagger}$} \\
\hline Normal (45-138\%), n (\%) & $16(47)$ \\
\hline Moderately reduced (10-45\%), n (\%) & $12(35)$ \\
\hline Severely reduced $(<10 \%), \mathrm{n}(\%)$ & $6(18)$ \\
\hline
\end{tabular}

At the time of neuropsychological evaluation, 10 (29\%) of 35 patients had suffered from recurrent TTP bouts. *Neuropsychological evaluation was performed at a median time of 36 months (IQR: 17-54) from the last acute TTP event. ${ }^{\dagger}$ Available in 33 (platelet count, hemoglobin and number of PEX to remission at first acute TTP episode),34 (ADAMTS13 activity close to neuropsychological evaluation) and 31 subjects (platelet count and hemoglobin close to the neuropsychological evaluation). IQR: interquartile range; PEX: plasma exchange; TTP: thrombotic thrombocytopenic purpura

malized scores than the Italian reference sample ${ }^{14}$ in all scales but physical activity. With regard to the physical components, the most impacted area was the physical role, with a mean score of 57 (median 55; range: 40-85) and 11 patients $(31 \%)$ with scores below 50 . With regard to the mental components, emotional role was the most compromised, with a mean score of 43 (median 43; range: 30-56) and 22 patients (63\%) with scores below 50. Overall, the mental dimension was more affected than the physical dimension, with the mental component score MCS-36 (equivalent to the sum of MH, ER, SF and VI scores) lower than the physical component score PCS36 (equivalent to the sum of PA, RP, BP and GH scores) by almost 60 points (mean difference -58.43 ; $95 \%$ CI: -71.49 $-45.37)$ and $15(43 \%$ of patients pertaining to the MCS36 ) versus four (11\% of patients pertaining to the PCS-36) patients with scores below 50, the commonly accepted cut-off for an acceptable quality of life. ${ }^{15,16}$

Finally, at correlation analysis, the better the mental component score of the HrQoL survey was, the better were the results of emotional wellbeing assessments, especially the anxiety evaluation (MCS-36 vs. HAM-A test: Spearman rho $-0.358, P=0.035$; MCS-36 vs. HAM-D test: Spearman rho $-0.316, P=0.064)$. 
Table 2. Descriptive statistics of neuropsychological tests in acquired thrombotic thrombocytopenic purpura patients and mean values of the Italian general population.

$\begin{array}{lccc}\text { ITest } & \text { MP patients } & \text { General population } & \text { Mean difiference } \\ & \text { Mean (SD) } & \text { Mean } & (95 \% \text { CI) }\end{array}$

Memory

$\begin{array}{lccc}\text { Digit span (direct) } & 5.74(1.12) & 7.00 & -1.26(-1.64,-0.87) \\ \text { Digit span (backword) } & 4.51(1.54) & 6.00 & -1.49(-2.02,-0.96) \\ \text { Rey word list (direct)* } & 26.37(7.74) & 32.20 & -5.87(-8.57,-3.17) \\ \text { Rey word list (deferred)* } & 4.03(1.86) & 5.70 & -1.67(-2.32,-1.02) \\ \text { Attention } & & & -10.63(-15.81,-5.44) \\ \text { Trail making A, seconds } & 34.37(15.1) & 45.00 & 65.09(47.23,82.94) \\ \text { Trail making B, seconds } & 214.09(51.99) & 149.00 & \text {. }\end{array}$

In memory tests a higher score indicates a better performance, in attention tests a lower score indicates a better performance. At the time of neuropsychological evaluation, 10 (29\%) of 35 patients had suffered from recurrent TTP bouts. *: Available in 34 TTP patients; CI: confidence interval; SD: standard deviation; TTP: thrombotic thrombocytopenic purpura.

Table 3. Descriptive statistics of neuropsychological tests in acquired thrombotic thrombocytopenic purpura (TTP) patients with and without neurological manifestations at onset of the first acute TTP event.

\begin{tabular}{|c|c|c|c|}
\hline İest & $\begin{array}{l}\text { Neurological invo } \\
\text { Present }(n=22)\end{array}$ & $\begin{array}{l}\text { acute TIP event } \\
\text { Absent }(n=13)\end{array}$ & Mean difference ( $95 \%$ Cl) \\
\hline \multicolumn{4}{|l|}{ Memory, mean (SD) } \\
\hline Digit span (direct) & $5.45(1.14)$ & $6.23(0.93)$ & $-0.78(-1.54,-0.02)$ \\
\hline Digit span (backword) & $4.18(1.59)$ & $5.08(1.32)$ & $-0.90(-1.96,0.17)$ \\
\hline Rey word list (direct)* & $25.36(7.73)$ & $27.89(7.80)$ & $-2.54(-8.11,3.04)$ \\
\hline Rey word list (deferred)* & $3.50(1.92)$ & $4.89(1.44)$ & $-1.39(-2.65,-0.13)$ \\
\hline \multicolumn{4}{|l|}{ Attention, mean (SD) } \\
\hline Trail making A, seconds & $37.18(16.26)$ & $29.62(12.00)$ & $7.57(-3.01,18.14)$ \\
\hline Trail making B, seconds & $207.82(49.91)$ & $224.69(55.71)$ & $-16.87(-53.95,20.20)$ \\
\hline
\end{tabular}

In memory tests a lower score indicates a worse performance, in attention tests a higher score indicates a better performance. At the time of neuropsychological evaluation, 9 of $22(41 \%)$ and 1 of $13(8 \%)$ patients with and without neurological involvement at the first acute TTP event had suffered from recurrent TTP bouts, respectively. ${ }^{*}$ : Available in 34 TTP patients; CI: confidence interval; SD: standard deviation; TTP: thrombotic thrombocytopenic purpura.

\section{Discussion}

Neurological signs and symptoms of acute TTP are mainly transient, brief and resolve with remission of the acute phase. Our study demonstrates persisting neurological, neuropsychological, emotional and HrOoL impairments in TTP patients even years after the acute phase.

During the remission phase, TTP patients showed a significant impairment in memory domains (direct, backward and deferred memory) when compared with the general population. This memory impairment was positively associated with the presence of neurological symptoms during the acute phase of the disease, as shown by the comparison between patients with and without neurological involvement during the first acute TTP event. Attention domains were also affected, but they were unrelated to neurological involvement during the acute phase. Our results are in line with previous findings by Kennedy et al. in 24 acquired TTP patients from the Oklahoma Registry, who performed significantly worse than the US reference population in both attention and memory functions. ${ }^{3,17}$ Conversely, at variance with our results, previous studies did not report an association of neurocognitive impairment with the occurrence of neurological manifestations at the time of the acute TTP event, ${ }^{3,18}$ although a trend towards a worse mental performance was detected in German patients with neurolog- ical symptoms compared with patients with no neurological symptoms (median of FLei mental performance score: 45 [IOR: 15-65] vs. 31 [IOR: 13-40], Mann-Whitney U test $P=0.193){ }^{18}$ It is worth-noticing that the prevalence of relapsing TTP cases were higher in patients with than without neurological symptoms during the first acute event ( $41 \%$ vs. $8 \%)$. Unfortunately, the low sample size did not allow us to discriminate the effects of these two factors.

Beside cognitive problems, we detected clinical anxiety and depression in $20 \%$ and $43 \%$ of interviewed patients. An even higher prevalence of depression symptoms in acquired TIP patients was reported in two US and one German cohorts $\left(59 \%,{ }^{17} 81 \%, 19\right.$ and $73 \%,{ }^{18}$ respectively), which included also cases of major depression $(29 \%, 18$ $37 \%,{ }^{19}$ and $\left.14 \%\right) .{ }^{18}$ However, a pre-existing diagnosis of depressive disorder was not an exclusion criterion in these studies, which may partly explain the differences in the observed prevalence. In our study, the results of anxiety and depression tests were negatively correlated with scores of neurocognitive assessments, indicating that patients with symptoms of psychologic distress also had more pronounced cognitive defects. This is consistent with the findings of Falter and colleagues ${ }^{18}$ who reported a strong correlation between an impaired mental performance and the severity of depression in 84 TTP patients.

It is interesting to compare our results with other cardio- 
Table 4. Descriptive statistics of neuropsychological tests in acquired thrombotic thrombocytopenic purpura patients with and without ADAMTS13 deficiency next to the psychological evaluation.

\begin{tabular}{cc} 
ADAMIIS13 activity during remission & Mean difierence (95\% CI) \\
\hline$<45 \%(n=18)$ & $\geq 45 \%(n=16)$
\end{tabular}

Memory, mean (SD)

Digit span (direct)

$\begin{array}{ccc}6.06(1.00) & 5.50(1.16) & 0.56(-0.20,1.31) \\ 4.61(1.79) & 4.50(1.27) & 0.11(-0.98,1.21) \\ 27.22(7.72) & 26.09(7.65) & 1.13(-4.33,6.59) \\ 4.44(1.67) & 3.73(2.02) & 0.72(-0.59,2.02)\end{array}$

$4.44(1.67)$

$3.73(2.02)$

$0.72(-0.59,2.02)$

Attention, mean (SD)

Trail making A, seconds

32.06 (13.39)

36.94 (17.32)

$-4.88(-15.62,5.87)$

Trail making B, seconds

$209.89(58.93)$

$220.94(45.19)$

$-11.05(-48.09,26.00)$

In memory tests a lower score indicates a worse performance, in attention tests a higher score indicates a better performance. At the time of neuropsychological evaluation, 5 of $18(28 \%)$ and 5 of $16(31 \%)$ patients with and without ADAMTS13 deficiency next to the visit had suffered from recurrent TTP bouts, respectively. ${ }^{*}$ :Available in 34 TTP patients; CI: confidence interval; SD: standard deviation.

Table 5. Descriptive statistics of health-related quality of life components in acquired thrombotic thrombocytopenic purpura patients and in Italian reference individuals.

\begin{tabular}{|c|c|c|c|c|}
\hline \multirow[t]{2}{*}{ T̄est } & \multicolumn{2}{|c|}{ TIP patients (n=35) } & \multirow{2}{*}{$\begin{array}{c}\text { General population } \\
(n=2031) \\
\text { mean (SD) }\end{array}$} & \multirow{2}{*}{$\begin{array}{l}\text { Mean difference } \\
\text { (95\%Cl) }\end{array}$} \\
\hline & mean (SD) & N with score<50 (\%) & & \\
\hline \multicolumn{5}{|l|}{ Physical domain } \\
\hline Physical activity & $81.40(15.37)$ & $2(6)$ & $84.46(23.18)$ & $-3.06(-8.43$ to 2.31$)$ \\
\hline Limitation physical role & $56.77(13.04)$ & $11(31)$ & $78.21(35.93)$ & $-21.44(-26.17$ to -16.72$)$ \\
\hline Pain & $62.20(13.92)$ & $5(14)$ & $73.67(27.65)$ & $-11.47(-16.39$ to -6.55$)$ \\
\hline General health & $59.91(11.54)$ & $5(14)$ & $65.22(22.18)$ & $-5.31(-9.38$ to -1.24$)$ \\
\hline PCS-36 & $260.29(44.63)$ & $4(11)^{*}$ & NA & NA \\
\hline \multicolumn{5}{|l|}{ Mental domain } \\
\hline Vitality & $52.46(12.85)$ & $13(37)$ & $61.89(20.69)$ & $-9.43(-13.93$ to -4.93$)$ \\
\hline Social activity & $54.26(8.60)$ & $7(20)$ & $77.43(23.34)$ & $-23.17(-26.28$ to -20.06$)$ \\
\hline Limitation emotional role & $42.69(8.76)$ & $22(63)$ & $76.16(37.25)$ & $-33.47(-36.86$ to -30.08$)$ \\
\hline Mental health & $52.46(5.45)$ & $4(11)$ & $66.59(20.89)$ & $-14.13(-16.20$ to -12.06$)$ \\
\hline MCS-36 & $201.86(23.84)$ & $15(43)^{*}$ & NA & NA \\
\hline
\end{tabular}

At the time of neuropsychological evaluation, 10 (29\%) of 35 patients had suffered from recurrent TTP bouts. *Being PCS-36 and MCS-36 the weighted sum of the original scales of the SF-36, this number indicates patients with a score below 200.CI: confidence interval; NA: not available; SD: standard deviation; MCS-36: mental component summary (MCS) of the Short Form (36) Health Status Questionnaire (SF36); PCS-36: physical component summary (PCS) of the Short Form (36) Health Status Questionnaire (SF36);TTP: thrombotic thrombocytopenic purpura.

vascular and neurovascular diseases. High percentage of cognitive impairment were also found in patients after acute coronary syndrome ( $16 \%$ of patients), ${ }^{20}$ stroke (onethird of the sample examined), ${ }^{21}$ and after a transient ischemic attack (TIA) (more than a third of patients). ${ }^{22}$ After TIA, also depressive symptoms were found as prevalent as $34 \% .{ }^{23}$ However, there are important differences between these studies and ours. First, the evaluation of cognitive decline and depression were generally made during the acute phase and in patients older than ours. ${ }^{23}$ Second, the literature mainly highlights a major deterioration at the level of functional abilities in daily life activities, especially in stroke patients. Finally, different tests were performed and different cognitive domains (e.g. language) evaluated, making any comparison difficult. We also found an overall impaired quality of life compared with the general population. In the HrQoL domains, mental components were more impaired than physical components, suggesting that the condition determines a considerable emotional burden, probably related to its course of intermittent relapse and remission phases. Indeed, the
HrQoL mental domain was negatively affected by the presence of clinical anxiety and depression in our patients. Our findings are consistent with those of Lewis et al. ${ }^{2}$ and Cataland et al. ${ }^{24}$ although they reported a greater impact of acquired TTP on the physical component of the HrQoL,2 and on both the mental and the physical component. ${ }^{24}$

We believe that our findings have clinical relevance for the management of TTP patients. Clinicians should be aware of the association between neurological manifestations during the acute TTP episodes and long-term impaired neuropsychological abilities. They should put attention on signs and symptoms of neurological impairment during the remission phase and consider early signs of anxiety and depression in order to improve the quality of life of TTP patients.

Our study has limitations. First, the neurocognitive and emotional status of the patients and their quality of life before their first episode of TTP was not objectively evaluated. However, patients did perceive and referred at the time of the psychological consultation a worsening of their conditions after ITP diagnosis. Second, the evalua- 
tion of neurological and cognitive functions abnormalities observed in TTP patients in the remission phase and far from the clinical manifestations of acute TTP were not supported by instrumental neuro-functional analysis such as functional magnetic resonance imaging, which might be needed to assess the aforementioned alterations at the organic level. Third, we included patients with a history of a single acute TTP event but also patients with relapsing TTP, rendering our study population heterogeneous (in order to exclude potential comorbidities only primary TTP patients should be evaluated). However, the occurrence of multiple TTP episodes was never found to be associated with neurocognitive, emotional or HrQoL assessments in any previous study. ${ }^{3,17,18,24}$ Fourth, the tests performed to evaluate clinical anxiety and depression, despite being well-validated tools, can only be suggestive of a psychiatric diagnosis, which indeed can only be confirmed by a proper psychiatric interview. Fifth, despite the relatively large sample size for a rare disease, numbers in some analyses are small, leading to statistical uncertainty and wide CI. This is a problem of any study in the field of rare diseases, and stimulate more efforts to promote collaboration between centers. Finally, the design of our study is cross-sectional and, thus cannot provide risk estimates and data about predictive factors. On the other hand, as far as we know, our study is the most comprehensive investigation on TTP patients, including clinical, cognitive, emotional and HrOoL assessment.

In conclusion, we demonstrated that, despite successful treatment with plasma exchange and immunosuppressive therapy in the acute stage of the disease, patients with TTP suffer from long term neurological sequelae even years after the acute phase. TTP patients with neurological involvement at the first acute episode seem to be at higher risk of developing memory dysfunction. Furthermore, TTP patients have increased levels of anxiety and depression, which negatively affect their quality of life. A faster improvement of the acute state of the disease by novel drugs might have a role in this process and further studies are required to solve this question.

\section{References}

1. Deford CC, Reese JA, Schwartz LH, et al. Multiple major morbidities and increased mortality during long-term follow-up after recovery from thrombotic thrombocytopenic purpura. Blood. 2013;122(12):2023-2029.

2. Lewis OF, Lanneau MS, Mathias SD, et al. Long-term deficits in health-related quality of life following recovery from thrombotic thrombocytopenic purpura. Transfusion. 2009;49(1):118-124.

3. Kennedy KA, Lewis OF, Scott JG, et al. Cognitive deficits after recovery from thrombotic thrombo- cytopenic purpura. Transfusion. 2009:49(6):1092-1101.

4. Ware Jr, JE, Sherbourne CD. The MOS 36item short-form health survey (SF-36): I. Conceptual framework and item selection. Med Care. 1992;30(6):473-483.

5. Rabin R, de Charro F. EQ-SD: a measure of health status from the EuroQol Group. Ann Med. 2001;35(5):337-343.

6. Wechsler S. The Semantic Basis of Argument Structure. New York, NY: CSLI Publications (Center for the Study of Language and Information). 1995, Stanford, Cambridge University Press.

7. Rey A. L'Examen clinique en psychologie. (The Clinical Exam in Psychology), 1964, Paris: Presses Universitaires de France.

8. Tombaugh TN. Trail Making Test A and B: normative data stratified by age and education. Arch Clin Neuropsychol. 2004; 19(2):203-214
9. Hamilton M. A rating scale for depression. J Neurol Neurosurg Psychiatry. 1960;23:5665.

10. Hamilton M. The assessment of anxiety states by rating. Br J Med Psychol. 1959;32:50-55.

11. Mondini S, Mapelli D, Vestri A, et al. Esame neuropsicologico breve. Milano: Raffaello Cortina Editore. 2003:160.

12. Carlesimo GA, Caltagirone C, Gainotti GU, et al. The mental deterioration battery: normative data, diagnostic reliability and qualitative analyses of cognitive impairment. Eur Neurol. 1996;36(6):378384.

13. Giovagnoli AR, Del Pesce M, Mascheroni $S$, et al. Trail making test: normative values from 287 normal adult controls. Ital J Neurol Sci. 1996;17(4):305-309.

14. Apolone G, Mosconi P. The Italian SF-36 Health Survey: translation, validation and norming. J Clin Epidemiol. 1998; 51(11):1025-1036.

15. Ware JE, Kosinski M, Gandek B, et al. The factor structure of the SF-36 Health Survey in 10 countries: Results from the IQOLA Project. J Clinic epidemiol. 1998; 51(11):1159-1165

16. Obidoa CA, Reisine SL, Cherniack M. How Does the SF-36 Perform in Healthy Populations? A Structured Review of Longitudinal Studies. J Soc Behav Health. 2010;4(1):30-48.

17. Han B, Page EE, Stewart LM, et al. Depression and cognitive impairment following recovery from thrombotic thrombocytopenic purpura. Am J Hematol. 2015;
90(8):709-714.

18. Falter T, Schmitt V, Herold S, et al Depression and cognitive deficits as longterm consequences of thrombotic thrombocytopenic purpura. Transfusion. 2017; 57(5):1152-1162.

19. Chaturvedi S, Oluwole O, Cataland S, et al. Post-traumatic stress disorder and depression in survivors of thrombotic thrombocytopenic purpura. Thromb Res. 2017;151:51-56.

20. Saczynski JS, McManus DD, Waring ME, et al. Change in cognitive function in the month after hospitalization for acute coronary syndromes: findings From TRACECORE (transition, risks, and actions in coronary events-center for outcomes research and education). Circ Cardiovasc Qual Outcomes. 2017;10(12)

21. Lisabeth LD, Sánchez BN, Baek J, et al Neurological, functional, and cognitive stroke outcomes in Mexican Americans. Stroke. 2014;45(4):1096-1101.

22. van Rooij FG, Kessels RP, Richard E, et al. Cognitive impairment in transient ischemic attack patients: a systematic review. Cerebrovasc Dis. 2016:42(1-2):1-9.

23. Mitchell AJ, Sheth B, Gill J, et al. Prevalence and predictors of post-stroke mood disorders: A meta-analysis and meta-regression of depression, anxiety and adjustment disorder. Gen Hosp Psychiatry. 2017;47:4860

24. Cataland SR, Scully MA, Paskavitz J, et al. Evidence of persistent neurologic injury following thrombotic thrombocytopenic purpura. Am J Hematol. 2011;86(1):87-89. 